\title{
Minimally invasive chevron/akin osteotomy: radiographic outcomes
}

\author{
Kepler Alencar Mendes de Carvalho' ${ }^{(\mathbb{D})}$, Fernando Garnica Torrico ${ }^{1}{ }^{\mathbb{D}}$, David Satiro Borges Pereira de Oliveira' ${ }^{\mathbb{D}}$, \\ André Donato Baptista' (D)
}

1. Nossa Senhora do Pari Beneficent Association, São Paulo, SP, Brazil.

\begin{abstract}
Objective: To evaluate radiographic outcomes in patients undergoing minimally invasive Chevron/Akin osteotomy (MICA) for hallux valgus correction.

Methods: We have treated 31 patients (40 feet). Preoperative and postoperative hallux metatarsophalangeal angles (hallux valgus angles, HVA), intermetatarsal angles (IMA) between the first and second metatarsals, and distal metatarsal articular angles (DMAA) were followed up for 12 months.

Results: The mean age of the patients was 53.2 years. Mean preoperative HVA, IMA, and DMAA values were $28.85^{\circ}, 15.38^{\circ}$, and $14.35^{\circ}$, respectively. The mean postoperative angles measured after 1 year were $10.60^{\circ}, 7.95^{\circ}$, and $7.48^{\circ}$. Improvements in HVA, IMA, and DMAA values showed statistical relevance. There were no losses in angular parameters during follow-up.

Conclusion: The radiographic outcomes in patients undergoing MICA osteotomy for hallux valgus correction followed up for 12 months showed significant improvements and no recurrence of the deformity. Our results suggest that this technique is effective for correcting hallux valgus.
\end{abstract}

Level of Evidence III; Therapeutic Studies; Comparative Retrospective Study.

Keywords: Hallux valgus/surgery; Osteotomy; Radiography; Minimally invasive surgical procedures.

\section{Introduction}

Hallux valgus is a common deformity of the forefoot, characterized by lateral hallux deviation and medial deviation of the first metatarsal. Its overall prevalence is $23 \%$ among individuals aged 18 to 65 years and $35 \%$ in the older population (> 65 years old). It mainly affects women, with a prevalence of $30 \%$, compared to $13 \%$ in men ${ }^{(1-3)}$.

Pain in the hallux metatarsophalangeal region is a quite common symptom, which may result in limitation of daily activities and impaired quality of life $\mathrm{e}^{(1,4-6)}$. Genetics is an important predictive factor for the disease, with nearly $68 \%$ of patients having familial inheritance. The use of inappropriate shoes, bone abnormalities, and excessive pronation of the foot are also among etiological factors ${ }^{(7,8)}$.
Conservative treatment consists in wearing adequate shoes with wide and deep anterior chambers and low heels to minimize the patient's pain and progressive deformity ${ }^{(7)}$.

In order to determine the severity of hallux valgus, we plotted the metatarsophalangeal angle (hallux valgus angle, HVA) and the intermetatarsal angle (IMA) on X-ray images. The HVA angle was plotted at the intersection of the lines along the long axis of the first metatarsal and the proximal phalanx of the hallux. The angle between the long axis of the first and second metatarsals was the IMA. Both angles were checked on anteroposterior radiographs of weight-bearing feet. The deformity was thus classified as: mild (HVA $>19^{\circ}$ and IMA $>13^{\circ}$ ), moderate (HVA between $20^{\circ}$ and $40^{\circ}$ and IMA between $14^{\circ}$ and $20^{\circ}$ ), and severe (HVA $>40^{\circ}$ and IMA >
Study performed at Nossa Senhora do Pari Beneficent Association, São Paulo, SP, Brazil.

Correspondence: Képler Alencar Mendes de Carvalho. Rua Hannemann, 234 Pari - 03031-040, São Paulo - SP, Brazil. E-mail: kepler.ortopedia@gmail.com. Conflicts of Interest: none. Source of funding: none. Date received: July 13 , 2021. Date accepted: July 22, 2021. Online: August 31, 2021.

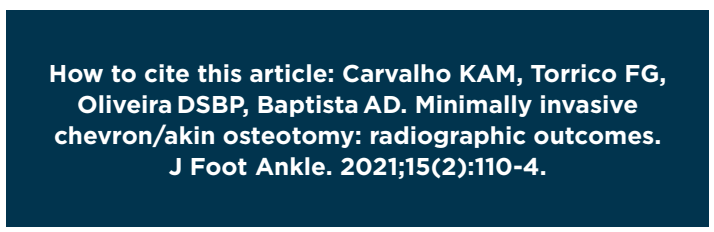


$\left.20^{\circ}\right)^{(7)}$. These radiographic angles have excellent correlations with clinical aspects ${ }^{(4,8)}$. The distal metatarsal articular angle (DMAA) is defined as the relationship between the distal articular surface and the longitudinal axis of the first metatarsal bone. The DMAA is considered a fundamental aspect to be evaluated while planning surgical corrections. To achieve total congruence of the metatarsophalangeal joint of the hallux, it is paramount to avoid or decrease the incidence of insufficient corrections or recurrences. In normal feet, this angle should not exceed $8^{\text {o( }(7,9)}$.

Minimally invasive surgery for correcting forefoot deformities has gained popularity worldwide in recent years. These techniques preserve soft tissues and reduce recovery time and morbidities associated with the disease process and surgical intervention ${ }^{(2,10,11)}$.

The distal Chevron metatarsal osteotomy, first described in 1962 , is a widely accepted technique used in the treatment of mild and moderate deformities. It shows satisfactory results in adult individuals of all age groups, promoting pain relief, restoration of function, and lasting correction of the deformity ${ }^{(4,10)}$. However, publications regarding its efficacy in severe deformities are still scarce in the literature ${ }^{(4)}$.

With the advent of minimally invasive techniques for correcting hallux valgus, Vernois idealized the minimally invasive Chevron/Akin osteotomy (MICA) osteotomy ${ }^{(12)}$. In this technique, owing to less damage to soft tissues and metatarsal head vascularization, it is possible to laterally translate the capital fragment through up to $100 \%$ of the osteotomy contact area. This increases the potential to correct severe deformities when compared to the traditional open technique. In some cases, the contact between the proximal and distal portions of the first metatarsal is made only by the cortical bone ${ }^{(4)}$. The osteotomy is fixed with cortical screws, providing sufficient stability for mild or aggressive corrections ${ }^{(2,10,12,13)}$.

\section{Methods}

This study was approved by the Research Ethics Committee registered at Plataforma Brasil under the Certificate of Presentation for Ethical Appreciation (CAAE number 35995820.5.0000.0068).

We operated on 31 patients ( 40 feet) using the MICA technique between June 2016 and January 2018. The exclusion criteria for patients in this study were peripheral sensitive neuropathy, symptomatic osteoarthritis with limited range of motion of the first metatarsophalangeal joint, and severe vascular disease.

\section{Radiographic analysis}

Pre- and postoperative weight-bearing anteroposterior (AP) view radiographs were performed. Radiographic data were recorded preoperatively and at the 6-month and 12-month follow-up visits. The analysis followed the trigonometric measurement model established for Chevron's osteotomy ${ }^{(14)}$. We evaluated the following radiographic parameters: HVA, DMAA, and IMA.

\section{Surgical procedure}

The surgical procedure consisted of a bunionectomy performed with $12 \times 3.1 \mathrm{~mm}$ Wedge cutters, followed by percutaneous Chevron osteotomies with $20 \times 2 \mathrm{~mm}$ Shannon burrs and Akin osteotomies performed with $12 \times 2 \mathrm{~mm}$ Shannon burrs. Osteotomy fixation was done using $23.5 \mathrm{~mm}$ fully threaded cannulated screws or $4.5 \mathrm{~mm}$ headless compression screws (Figure 1). All surgical steps were performed under fluoroscopic control.

\section{Results}

We included 31 patients (40 feet) operated on using the MICA technique for hallux valgus correction. The mean age of the patients was 53.45 ( \pm 2.25 ) years (ranging from 23 to 77 years) and $90 \%$ of them were women.

The mean preoperative HVA angle was $28.85^{\circ}\left( \pm 10.42^{\circ}\right.$ ). At the 6-month postoperative follow-up, the mean value was $10.78^{\circ}\left( \pm 6.93^{\circ}\right)$, and at the $12-$ month follow-up, it was $10.60^{\circ}$ $\left( \pm 6.65^{\circ}\right)$ (Figure 2).

The mean preoperative IMA value was $15.38^{\circ}\left( \pm 4.07^{\circ}\right)$. At 6 months postoperative, the mean value was $8.11^{\circ}\left( \pm 2.60^{\circ}\right)$, and after 12 months, it was $7.95^{\circ}\left( \pm 2.80^{\circ}\right.$ ) (Figure 3 ).

Regarding the DMAA, the mean preoperative value was $14.35^{\circ}\left( \pm 6.83^{\circ}\right)$, after 6 months it was $7.75^{\circ}\left( \pm 5.51^{\circ}\right)$, and after 12 months, $7.48^{\circ}\left( \pm 5.39^{\circ}\right.$ ) (Figure 4$)$.

As sample distributions for the HVA, DMAA, and IMA were not symmetrical, we opted for the Friedman test in our initial statistical evaluation. The test analyzed 3 paired segments (preoperative vs 6-month postoperative vs 12 -month postoperative) to determine the statistically significant difference $(p<0.001)$ between preoperative and postoperative measurements (Table 1).

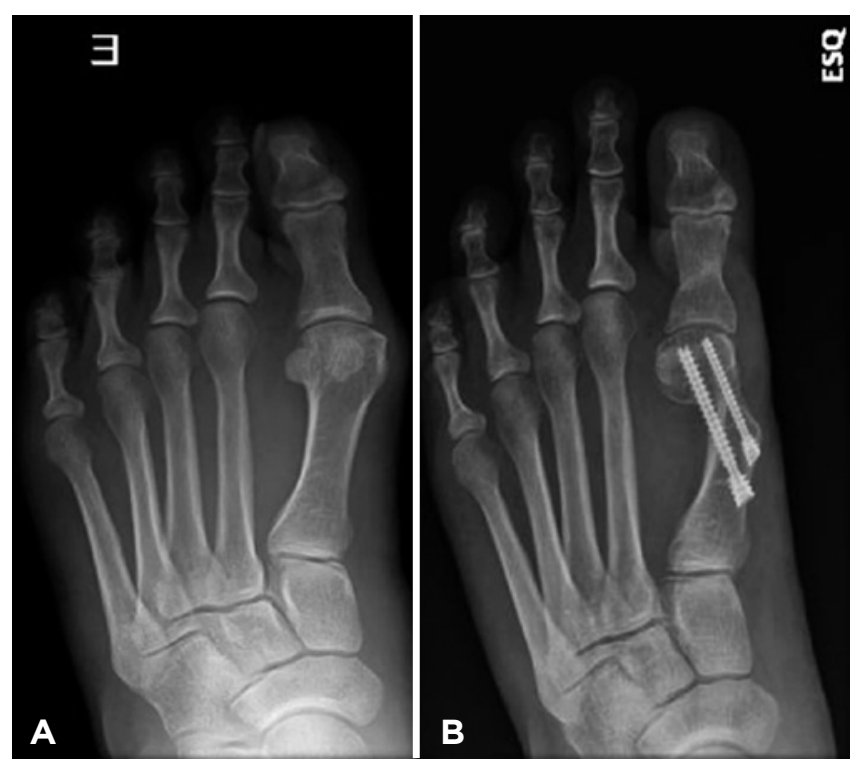

Figure 1. A) Preoperative radiographic image. B) Radiographic image 12 months after surgery. 


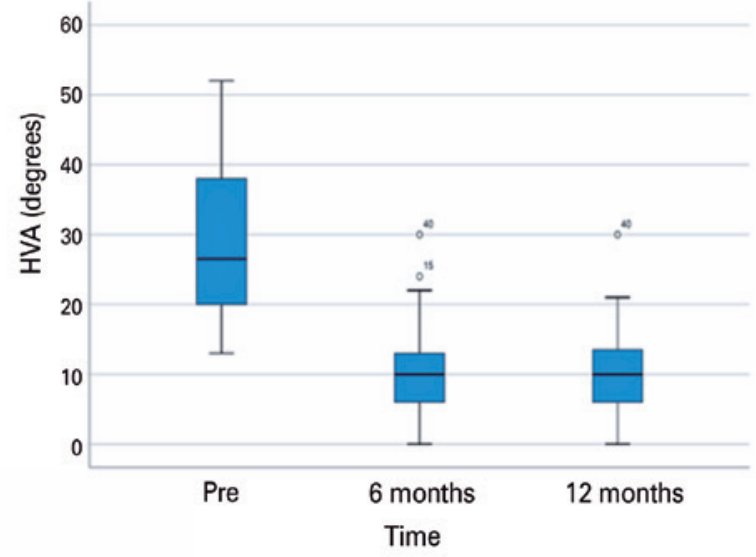

Figure 2. Radiographic parameters over time. Metatarsophalangeal angle (hallux valgus angle, HVA), (mean/standard deviation, in degrees).

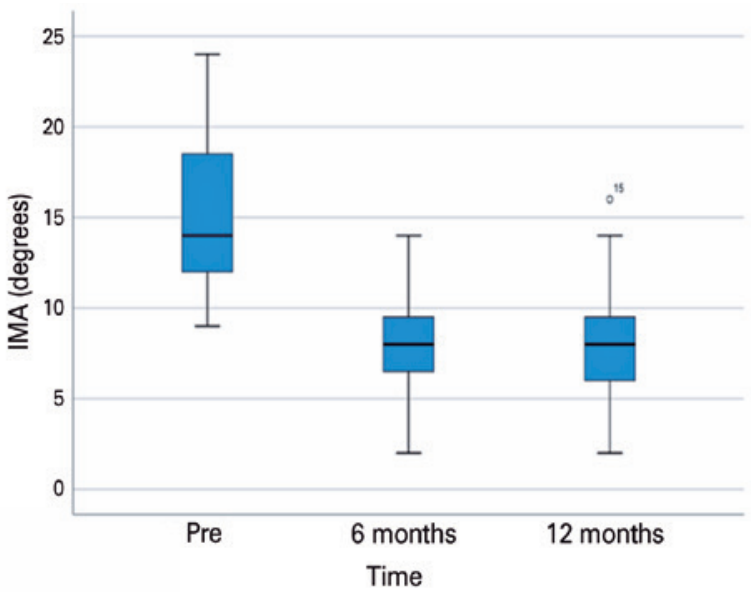

Figure 3. Radiographic parameters over time. Intermetatarsal angle (IMA) (mean/standard deviation, in degrees).

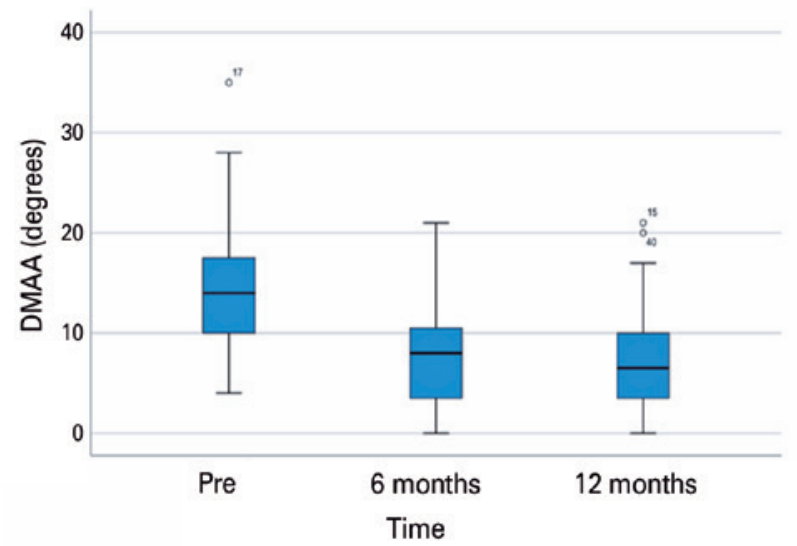

Figure 4. Radiographic parameters over time. Distal metatarsal articular angle (DMAA) (mean/standard deviation, in degrees).
Table 1. Friedman tests for 3 paired follow-ups (preoperative [pre-op] vs 6 -month postoperative [post-op] vs 12-month postoperative).

\begin{tabular}{lccc} 
& HVA & IMA & DMAA \\
\cline { 2 - 4 } & (Pre-op vs 6 & months post-op vs & 12 \\
\hline N & 40 & 40 & 40 \\
Chi-squared & 72.788 & 69.664 & 35.586 \\
p value & $\mathrm{p}<0.001$ & $\mathrm{p}<0.001$ & $\mathrm{p}<0.001$ \\
\hline DMAA: distal metatarsal articular angle; HVA: hallux valgus angle; IMA: intermetatarsal angle
\end{tabular}

Subsequently, the Wilcoxon test (matched pairs) was applied to verify at which point during the follow-up the statistically significant difference was found. We observed no differences in the HVA, DMAA, and IMA radiographic parameters from the 6-month to the 12-month follow-up $(p=0.788)$. On the other hand, HVA, DMAA, and IMA improved significantly when comparing the preoperative values to the 6 -month $(p<0.001)$ and 12 -month postoperative periods $(p<0.001)$ (Table 2).

\section{Discussion}

There is an increasing interest in minimally invasive surgery techniques for the treatment of forefoot pathologies, especially hallux valgus. Some of the reported advantages include less aggression to soft tissues and vascularization ${ }^{(5,6)}$. However, there is still no consensus in the medical literature on the most effective technique to correct hallux valgus ${ }^{(6)}$.

At our institution, the MICA osteotomy is the treatment of choice for most cases of mild, moderate, and severe hallux valgus deformities. We designed this study to assess radiographic parameters of patients undergoing this procedure and its effectiveness in the correction of hallux valgus deformities.

Following the trigonometric measurement model designed for Chevron's osteotomy ${ }^{(14)}$, we assessed the following radiographic parameters: HVA, DMAA, and IMA between the first and second metatarsal.

The HVA is the angle formed between the line running along the diaphyseal axis of the proximal phalanx of the hallux and the mechanical axis of the first metatarsal. We considered values below $15^{\circ}$ to be normal(7,14,15). We recorded a mean preoperative HVA of $28.8^{\circ}$. At the 12-month postoperative evaluation, the mean value decreased to 10.6 degrees, denoting a statistically significant difference $(p<0.01)$ and demonstrating a return of the HVA value to normal parameters.

The IMA is the angle formed between the lines that correspond to the first and second metatarsal axes. We considered normal values to be equal to or lower than $9^{\circ(7,14,15)}$. In our study, we found a mean preoperative IMA of $15.38^{\circ}$, and at the 12-month postoperative follow-up, the mean value decreased to $7.95^{\circ}$, denoting a statistically significant difference $(p<0.01)$ and return of the IMA to normal angular parameters. 
Table 2. Wilcoxon test assessing at what point during follow-up the statistically significant difference was found.

\begin{tabular}{|c|c|c|c|c|c|c|c|c|c|}
\hline & \multicolumn{3}{|c|}{ Metatarsophalangeal angle } & \multicolumn{3}{|c|}{ Intermetatarsal angle } & \multicolumn{3}{|c|}{ Distal metatarsal articular angle } \\
\hline & $\begin{array}{c}\text { Pre-op vs } \\
\text { P0 6m }\end{array}$ & $\begin{array}{l}\text { PO6m vs } \\
\text { P012m }\end{array}$ & $\begin{array}{c}\text { Pre-op vs } \\
\text { P0 12m }\end{array}$ & $\begin{array}{c}\text { Pre-op vs } \\
\text { P06m }\end{array}$ & $\begin{array}{c}\text { POGm vs } \\
\text { PO12m }\end{array}$ & $\begin{array}{c}\text { Pre-op vs } \\
\text { P012m }\end{array}$ & $\begin{array}{c}\text { Pre-op vs } \\
\text { P06m }\end{array}$ & $\begin{array}{l}\text { P06m vs } \\
\text { P0 12m }\end{array}$ & $\begin{array}{c}\text { Pre-op vs } \\
\text { P0 12m }\end{array}$ \\
\hline Z & $-5.514^{b}$ & $-0.199^{b}$ & $-5.513^{b}$ & $-5.389^{b}$ & $-0.269^{b}$ & $-5.519^{b}$ & $-4.497^{b}$ & $-1.550^{b}$ & $-4.591^{b}$ \\
\hline$p$ value & $<0.001$ & 0.842 & $<0.001$ & $<0.001$ & 0.788 & $<0.001$ & $<0.001$ & 0.121 & $<0.001$ \\
\hline
\end{tabular}

b. Based on positive posts.

Pre-op: preoperative; PO: postoperative; m: months.

Although the literature is still scarce about this theme, in a similar study evaluating HVA and IMA for this type of osteoto$m y^{(16)}$, the authors found similar results for angular corrections comparing preoperative and postoperative measurements.

The DMAA represents the orientation of the articular surface of the first metatarsal head(17), measured between the line connecting the 2 extreme points of the distal articular surface of the first metatarsal bone and the line perpendicular to its diaphyseal axis. This angle should not exceed $8^{\circ}$. Although some authors suggest that an altered DMAA may be a secondary radiographic projection of a pronation deformity, this measure is still widely used and influences treatment decision making ${ }^{(7,17-19)}$. Osteotomies that produce lateral rotation in the axial plane can potentially worsen the DMMA, requiring additional procedures for its correction. As the percutaneous Chevron is a distal translational osteotomy, we did not observe this deleterious effect. To our knowledge, there are no studies to date that explain etiologic factors for increased DMAA in hallux valgus.

In most cases, the progression of hallux valgus is simultaneous with an unstable first tarsometatarsal joint, which causes medial deviation and pronation of the first metatarsal(17).

We recorded a mean preoperative DMMA of $14.35^{\circ}$. At the 12 -month postoperative evaluation, this mean value decrea- sed to $7^{\circ}$, denoting a statistically significant difference $(p<0.01)$ and a return of the DMAA value to normal parameters. We did not intentionally care for the metatarsal pronation during the procedure, but we believe the lateral shift of the first metatarsal head itself promotes its correction.

This study's weaknesses include the small number of patients, its retrospective and non-randomized design, and the absence of clinical data. Although 2 experienced orthopedic surgeons performed all radiographic measurements, an unknown bias can affect the results of the study. In addition, the short follow-up and small sample group may be insufficient for us to reach relevant conclusions.

\section{Conclusion}

Many studies have suggested that hallux valgus is a three-dimensional deformity. Therefore, two-dimensional radiographic measurements may be insufficient to explain the complex nature of this deformity. The advent of three-dimensional imaging technologies, such as weight-bearing computed tomography scans, should bring us more accurate information about this complex deformity. Nevertheless, the radiographic parameters evaluated in this study showed that the MICA technique is effective in promoting satisfactory angular corrections that lasted for the 12-month follow-up.

Authors' contributions: Each author contributed individually and significantly to the development of this article: KAMC *(https://orcid.org/OOOO-OOO31082-6490) Conceived and planned the activities that led to the study, performed the surgeries, interpreted the results of the study, participated in the review process and approved the final version; FGT *(https://orcid.org/O000-0002-2127-6650) Data collection and interpreted the results of the study; DSBPO *(https://orcid.org/0000-0002-0201-9413) Data collection and interpreted the results of the study; ADB *(https://orcid.org/0000-0002-5991-1701) Performed the surgeries and approved the final version. All authors read and approved the final manuscript. *ORCID (Open Researcher and Contributor ID) (iD).

\section{References}

1. van Groningen B, van der Steen MC, Reijman M, Bos J, Hendriks JG. Outcomes in chevron osteotomy for Hallux Valgus in a large cohort. Foot (Edinb). 2016;29:18-24.

2. Silva RF, Baptista AD. Surgical treatment of moderate and severe hallux valgus: 30 cases undergoing surgery using the percutaneous Chevron technique. Sci J Foot Ankle. 2018;12(1):61-7.

3. Maffulli N, Longo UG, Marinozzi A, Denaro V. Hallux valgus: effectiveness and safety of minimally invasive surgery. A systematic review. Br Med Bull. 2011;97:149-67.
4. Lee KM, Ahn S, Chung CY, Sung KH, Park MS. Reliability and relationship of radiographic measurements in hallux valgus. Clin Orthop Relat Res. 2012;470(9):2613-21.

5. Palmanovich E, Myerson MS. Correction of moderate and severe hallux valgus deformity with a distal metatarsal osteotomy using an intramedullary plate. Foot Ankle Clin. 2014;19(2):191-201.

6. Cassinelli SJ, Herman R, Harris TG. Distal Metatarsal Osteotomy for Moderate to Severe Hallux Valgus. Foot Ankle Int. 2016;37(10):1137-45

7. Nery CAS. Hallux valgus. Rev Bras Ortop. 2001;36(6):183-200. 
8. Cohen JC, Richardson G, Fernandes RMP. Surgical treatment of moderate to severe hallux valgus with distal chevron osteotomy associated with distal soft tissue release. Rev ABTPé. 2010;4(2): 97-106.

9. Nery CAS, Apostólico Netto A, Benevento M, Réssio C. Radiographic Evaluation of the Hallux Valgus: Population Study of New Angular Parameters. Acta Ortop Bras. 2001;9(2):41-8.

10. Brogan K, Voller T, Gee C, Borbely T, Palmer S. Third-generation minimally invasive correction of hallux valgus: technique and early outcomes. Int Orthop. 2014;38(10):2115-21.

11. Jowett CRJ, Bedi HS. Preliminary Results and Learning Curve of the Minimally Invasive Chevron Akin Operation for Hallux Valgus. J Foot Ankle Surg. 2017;56(3):445-52.

12. Vernois J, Redfern DJ. Percutaneous Surgery for Severe Hallux Valgus. Foot Ankle Clin. 2016;21(3):479-93.

13. Redfern D, Perera AM. Minimally invasive osteotomies. Foot Ankle Clin. 2014;19(2):181-9.
14. Nery CAS. [Chevron osteotomy for the treatment of hallux valgus Part 1 - Clinical-radiological and trigonometric evaluation]. Rev Bras Ortop. 1995;30(6):385-92.

15. Hardy RH, Clapham JC. Observations on hallux valgus; based on a controlled series. J Bone Joint Surg Br. 1951;33(3):376-91.

16. Lee $M$, Walsh J, Smith MM, Ling J, Wines A, Lam P. Hallux Valgus Correction Comparing Percutaneous Chevron/Akin (PECA) and Open Scarf/Akin Osteotomies. Foot Ankle Int. 2017;38(8):838-46.

17. Lee SY, Chung CY, Park MS, Sung KH, Ahmed S, Koo S, Kang DW, Lee KM. Radiographic Measurements Associated With the Natural Progression of the Hallux Valgus During at Least 2 Years of Followup. Foot Ankle Int. 2018;39(4):463-70.

18. Coughlin MJ, Carlson RE. Treatment of hallux valgus with an increased distal metatarsal articular angle: evaluation of double and triple first ray osteotomies. Foot Ankle Int. 1999;20(12):762-70.

19. Lee KM, Ahn S, Chung $\mathrm{CY}$, Sung $\mathrm{KH}$, Park MS. Reliability and relationship of radiographic measurements in hallux valgus. Clin Orthop Relat Res. 2012;470(9):2613-21. 